ISSN 2447-9071

doi $10.36414 /$ rbmc.v6i14.32
Contato para correspondência: Rogério José de Almeida

E-mail:

rogerio.almeida@faculdadepm. edu.br

Conflito de interesse: Não

Financiamento: Recursos próprios

Recebido: 09/01/2020

Aprovado: 04/02/2020

\section{Preditores psicossociais da função sexual em mulheres em idade fértil atendidas em serviço de ginecologia}

\author{
Psychosocial predictors of sexual function in women of \\ childbearing age attended at gynecology service
}

Lara Silva Paixão', Malu Menezes Gomides' , Gabriela Cunha Fialho Cantarelli Bastos', Alessandra Arantes da Silva Campos ${ }^{1}$, Antonio Márcio Teodoro Cordeiro Silva ${ }^{1,2}$, Rogério José de Almeida ${ }^{1,2}$

\section{${ }^{1}$ Pontifícia Universidade Católica de Goiás - PUC Goiás \\ ${ }^{2}$ Faculdade da Polícia Militar - FPM}

\section{Resumo}

Este estudo teve por objetivo analisar os preditores psicossociais relacionados com a função sexual de mulheres em idade fértil atendidas em um serviço de ginecologia. Trata-se de um estudo transversal analítico com abordagem quantitativa desenvolvido por meio de aplicação de questionários às mulheres atendidas no serviço de ginecologia de uma clínica particular situada na cidade de Goiânia/ GO, atendidas no mês de julho e agosto de 2017, com faixa etária dos 18 a 49 anos e vida sexual ativa. Foramaplicados dois questionários, sendo um com questões psicossociais eo outroo Quociente Sexual - Feminino (QS-F), que avalia o desempenho sexual. Foi adotado um nível de significância de 5\% ( $p$ $<0,05)$ nas análises estatísticas realizadas a través do BioEstat 4.0. Foram entrevistadas 68 mulheres, idade média de 32,4 anos, sendo 68,6\% casada ou com união estável. Analisando escala QS-Fobteve uma média de 71,2 pontos, correspondente a um desempenhosexual regular abom. Foidemonstrado que as mulheres solteiras apresentaram função sexual significativamente superior em relação às casadas ( $p=0,048$ ). Em relação aos aspectos socioculturais, maiores escores foram encontrados nas mulheres que frequentemente iam a bares e festas $(p=0,024)$, que frequentemente realizavam algum tipo de atividade artística $(p=0,046)$ eque afirmaram frequentemente realizavam atividades turísticas $(p=0,022)$. Quanto aos aspectos pessoais, maiores escores foram encontrados nas mulheres não se consideravam tristes $(p=0,001)$, nas quefaziam ingestão deálcool $(p=0,039)$ enas que referiramestar satisfeitas com o próprio corpo $(p=0,031)$. A disfunção sexual feminina éum importante problema de saúdepúblicamundiale inúmeros fatores podem influenciar em sua ocorrência. Napresentepesquisa, os preditores psicossociais mostraram-se relevantes no papel da função sexual da mulher.

Palavras-Chave: Impacto psicossocial; Saúde sexual, Saúde da mulher.

\begin{abstract}
This study aimed to analyze the psychosocial predictors related to sexual function of women of childbearing age attending a gynecology service. This is a cross-sectional analytical study with a quantitative approach developed by applying questionnaires to women attending the gynecology service of a private clinic located in the city of Goiânia/GO, attended in July and August 2017, with age range. from 18 to 49 years old and active sex life. Two questionnaires were applied, one with psychosocial questions and the other the Sexual - Female Quotient (QS-F) that assesses sexual performance. A significance level of $5 \%(p<0.05)$ was adopted in the statistical analyzes performed using BioEstat 4.0 software. Sixty eight women were interviewed, with average age of 32.4 years, being $68.6 \%$ married or in a stable relationship. Analyzing QS-F scale obtained an average of 71.2 points, corresponding to a regular to good sexual performance. Single women were shown to have significantly higher sexual
\end{abstract}


function than married women ( $p=0.048$ ). Regarding sociocultural aspects, higher scores were found in women who often went to bars and parties ( $p=0.024)$, who often performed some kind of artistic activity $(p=0.046)$ and who said they often performed tourist activities $(p=0.022)$. Regarding personal aspects, higher scores were found in women who did not consider themselves sad ( $p=0.001$ ), those who were drinking alcohol $(p=0.039)$ and those who reported being satisfied with their bodies ( $p=$ 0.031 ). Female sexual dysfunction is a major worldwide public health problem and numerous factors can influence its occurrence. In the present research psychosocial predictors were relevant in the role of women's sexual function.

Keywords: Psychosocial impact; Sexual health, Women's health.

\section{Introdução}

O termo sexualidade vai além dos condicionantes biológicos e dos processos fisiológicos do corpo humano, já que abrange também as dimensões psicológicas, sociais, culturais e espirituais do indivíduo'. Chega-se na contemporaneidade com a perspectiva de que as mulheres, independentemente da faixa etária, praticarem relação sexual representa um ato de vivenciar o prazer².

Do ponto de vista biológico, a função sexual feminina caracteriza-se pela resposta sexual controlada à interação equilibrada de todas as partes do sistema nervoso (simpático e parassimpático), além de envolver fatores psicológicos e somáticos3. O ciclo sexual é responsável pela resposta sexual, que consiste em uma sequência temporal e coordenada composta por quatro etapas (desejo, excitação, orgasmo e resolução), cada qual com a sua neurofisiologia própria. 0 sistema nervoso parassimpático regula o aumento do fluxo sanguíneo durante a excitação sexual, enquanto o sistema nervoso simpático controla principalmente o orgasmo ${ }^{3}$.

Todavia, diversos são os fatores que interferem negativamente na atividade sexual humana, desencadeando as disfunções sexuais e promovendo sofrimento psíquico e dificuldades nos relacionamentos interpessoais. A disfunção sexual feminina refere-se à alteração do interesse pela atividade sexual. Soma-se a isso à dificuldade em vivenciar a excitação (subjetiva e/ou genital), em desencadear o desejo durante o envolvimento, à disfunção do orgasmo, dor à relação sexual, bem como à impossibilidade de relaxamento vaginal ${ }^{4}$.

A disfunção sexual manifesta-se de forma persistente ou recorrente, levando-se em consideração alguns critérios, tais como: idade, experiência do indivíduo, frequência e cronicidade do sintoma, sofrimento subjetivo e efeito sobre outras áreas do corpo. $O$ diagnóstico clínico é baseado na anamnese e exame ginecológico. Contudo, ainda que haja alteração da resposta sexual comprovada por causa orgânica, é diagnos- ticada disfunção sexual somente se a paciente apresentar insatisfação frente à sua condição sexual e se tiver o mínimo de seis meses de sintomatologia ${ }^{5}$.

A avaliação da disfunção sexual inclui fatores predisponen $\neg$ tes, precipitantes, mantenedores e contextuais. Tais fatores podem ser constitucionais ou por experiências prévias, caracterizando-se pela repressão à autonomia e à participação ativa e segura nos relacionamentos. Os precipitantes estão relacionados às condições de vida que podem desencadear disfunção sexual nas pessoas mais vulneráveis, como divórcio e separação conflituosa, experiências sexuais não satisfatórias, acidentes ou cirurgia mutiladora. Os mantenedores são aqueles responsáveis por transformar fracassos sexuais eventuais em dificuldades mais sérias. Por fim, os contextuais são os fatores estressores, como problemas financeiros graves, desemprego, exigências do parceiro, familiares, dentre outros ${ }^{4}$.

Sendo assim, o presente estudo objetiva o entendimento da função sexual específico das mulheres em idade fértil. A cultura ainda apoia uma educação que desestimula a mulher a conectar-se às sensações corporais, podendo gerar vergonha dos órgãos genitais e um desconhecimento sobre esses. Julgamentos de inadequação, vergonha, culpa e ansiedade podem monopolizar os pensamentos da mulher e interferir no aproveitamento da sexualidade 4 .

Nesse sentido, o foco da análise situa-se na perspectiva de mulheres em relação a sua função sexual. Assim, este estudo teve por objetivo analisar os preditores psicossociais relacionados com a função sexual de mulheres em idade fértil atendidas em um serviço de ginecologia.

\section{Métodos}

Trata-se de um estudo transversal descritivo com abordagem quantitativa. Este é um método de pesquisa no qual o fator e efeito são observados no mesmo intervalo de tempo 
analisado, portanto, um corte instantâneo na amostragem da população estudada. Além disso, busca fazer uma associação das peculiaridades individuais, tais como os fatores: sexo, idade, etnia, condições socioeconômicas, com o efeito ou a doença ${ }^{6}$.

A pesquisa foi realizada por meio de aplicação de questionários às mulheres atendidas no serviço de ginecologia de uma clínica particular situada na cidade de Goiânia/GO. Para a realização do cálculo amostral foi utilizado o número de pacientes atendidas nos últimos dois meses (julho e agosto de 2017) que antecederam a coleta de dados. Nesse período foram atendidas, dentro dos critérios de inclusão, um total de 81 mulheres. Com esta população utilizou-se um nível de confiança de $95 \%$ e margem de erro de 5\%, com a amostra final composta por 68 pacientes, sendo utilizada uma amostragem por conveniência.

A obtenção dos consentimentos e a coleta de dados ocorreram entre os meses de setembro e novembro 2017. Como critérios de inclusão foram pesquisadas mulheres na faixa etária dos 18 a 49 anos e vida sexual ativa. Foram excluídas as mulheres que responderam estar apresentando sintomas do climatério, que tinham diagnóstico de algum transtorno psiquiátrico, que tinham alguma patologia que interfira na função sexual e aquelas que estavam grávidas.

Foi utilizado um questionário sociodemográfico e de saúde construído e testado pelos pesquisadores em que se identificava dados como: idade, cor/etnia, estado civil, religião, idade da sexarca, número de relações sexuais na semana, atividades de lazer, dentre outras variáveis.

Foi utilizado o instrumento Quociente Sexual Feminino (QS-F), que tem por objetivo avaliar a função sexual das mulheres brasileiras. O QS-F foi desenvolvido no Programa de Estudos em Sexualidade (ProSex) do Instituto de Psiquiatria do Hospital das Clínicas da Faculdade de Medicina da Universidade de São Paulo7.

O QS-F é constituído por dez questões que avalia todas as fases do ciclo de resposta sexual e contempla os domínios: a) desejo e interesse sexual (questões, 1, 2 e 8); b) preliminares (questão 3); c) excitação pessoal e sintonia com o parceiro (questões 4 e 5); d) conforto (questões 6 e 7); e) orgasmo e satisfação (questões 9 e 10).

É uma escala que avalia o desempenho e a satisfação sexual feminina, de forma geral (pela soma dos escores de todas as questões). Avalia também, isoladamente, domínio por domínio desta atividade, pela consideração individualizada das questões pertinentes aos diferentes aspectos investigados $^{7}$. Cada questão é respondida numa escala que varia de zero a cinco pontos e o escore obtido é multiplicado por dois, resultando numa soma entre zero e 100. Na sétima questão, o valor da resposta ( 0 a 5 ) deve ser subtraído de 5 para se ter o escore final dessa questão.

Os valores maiores indicam melhor desempenho/satisfação sexual conforme se seguem:82-100 pontos: bom a excelente; $62-80$ pontos: regular a bom; $42-60$ pontos: desfavorável a regular; $22-40$ pontos: ruim a desfavorável; 0-20 pontos: nulo a ruim ${ }^{7}$.

As análises estatísticas foram realizadas com o auxílio pacote estatístico BioEstat 4.0. A caracterização do perfil sociodemográfico foi realizado por meio de frequência absoluta (n) e relativa (\%). A normalidade dos dados foi verificada utilizando o teste de D'Agostino-Pearson. Para as variáveis que foram constatadas a normalidade foram utilizados os testes de te de ANOVA. Já para as que não apresentaram normalidade utilizaram-se os testes de Mann-Whitney e Kruskal-Wallis. Em todas as análises foi adotado um nível de significância de $5 \%(p<0,05)$.

O estudo foi aprovado pelo Comitê de Ética em Pesquisa (CEP) da Pontifícia Universidade Católica de Goiás (PUC Goiás), sob o parecer no 2.128.407, CAAE: 69150217.2.0000.0037. Dessa forma, todos os participantes assinaram ao Termo de Consentimento Livre e Esclarecido.

\section{Resultados}

Foram entrevistadas 68 mulheres atendidas em serviço de Ginecologia de uma clínica particular situada na cidade de Goiânia/GO. A idade média das participantes foi de 32,4 anos $( \pm 7,6)$, sendo a maioria casada ou com união estável $(68,6 \%)$, de cor branca $(55,9 \%)$, com ensino superior completo ou incompleto $(80,9 \%)$, professavam religião $(83,8 \%)$ e forte envolvimento com a Igreja $(54,4 \%)$ (Tabela 1$)$.

Tabela 1. Comparação da função sexual com os aspectos demográficos das 68 mulheres atendidas em serviço de ginecologia, Goiânia/GO, 2018.

\begin{tabular}{lccccc}
\hline Variáveis & $\boldsymbol{n}$ & $\boldsymbol{f}(\%)$ & Média & $\boldsymbol{D P}$ & $\boldsymbol{p}$-valor \\
\hline Idade (anos) & 34 & 50,0 & 69,8 & 15,6 & \\
$18-32$ & 34 & 50,0 & 72,5 & 15,5 & 0,476 \\
$33-49$ & 32,4 & 7,6 & & & \\
Média (DP) & & & & & \\
Estado Civil & 40 & 58,8 & 68,6 & 16,6 & \\
Casada/União Estável & 28 & 41,2 & 74,9 & 13,3 & $0,048^{*}$ \\
Solteira & & & & & \\
Cor ou Etnia autorreferida & & & & & \\
Branca & 38 & 55,9 & 72,3 & 10,6 &
\end{tabular}




$\begin{array}{llllll}\text { Parda } & 21 & 30,9 & 70,9 & 19,8 & \\ \text { Outras } & 9 & 13,2 & 67,1 & 22,2 & 0,937\end{array}$

\section{Escolaridade}

$\begin{array}{lccccc}\text { Até } 9 \text { anos de estudos } & 2 & 2,9 & 72,0 & 8,5 & \\ \text { De } 10 \text { a } 20 \text { anos de estudos } & 11 & 16,2 & 75,5 & 13,9 & \\ \begin{array}{l}\text { Superior completo/incompleto } \\ \text { Religião }\end{array} & 55 & 80,9 & 70,3 & 16,0 & 0,613 \\ \text { Não } & & & & & \\ \text { Sim } & 11 & 16,2 & 74,5 & 9,7 & \\ & 57 & 83,8 & 70,5 & 16,4 & 0,822\end{array}$

Grau de Envolvimento com a Igreja

$\begin{array}{lccccc}\text { Forte } & 37 & 54,4 & 72,3 & 15,5 & \\ \text { Fraco } & 18 & 26,5 & 66,4 & 18,5 & \\ \text { Nenhum } & 2 & 2,9 & 74,0 & 11,3 & 0,551 \\ \text { Não Responderam } & 11 & 16,2 & & & \end{array}$

Renda Pessoal

$\begin{array}{lccccc}\text { Abaixo de um salário mínimo } & 5 & 7,4 & 84,4 & 7,9 & \\ \text { De 1 a 3 salários mínimos } & 30 & 44,1 & 67,7 & 15,7 & \\ \text { De 4 a } 6 \text { salários mínimos } & 15 & 22,1 & 69,3 & 20,2 & \\ \text { Acima de } 6 \text { salários mínimos } & 18 & 26,5 & 74,8 & 9,5 & 0,064\end{array}$

Mora

$\begin{array}{lccccc}\text { Sozinha } & 4 & 5,9 & 73,0 & 5,3 & \\ \text { Companheiro } & 18 & 26,5 & 68,0 & 15,2 & \\ \text { Filhos } & 4 & 5,9 & 75,0 & 16,7 & \\ \text { Pais } & 17 & 25,0 & 77,8 & 10,2 & \\ \text { Família } & 25 & 36,8 & 68,1 & 18,7 & 0,279\end{array}$

Tem Companheiro

\begin{tabular}{llllll} 
Não & 17 & 25,0 & 74,6 & 9,9 & \\
$\operatorname{Sim}$ & 51 & 75,0 & 70,0 & 16,9 & 0,466 \\
\hline
\end{tabular}

Analisando a função sexual feminina por meio da escala QS-F, obteve-se uma média do escore total de 71,2 pontos $( \pm 15,5)$, o qual corresponde a um desempenho/satisfação sexual regular a bom. Este mesmo desempenho correspondeu a $52,9 \%$ das entrevistadas desta pesquisa. Do restante, $26,5 \%$ obtiveram desempenho bom a excelente; $14,7 \%$ desfavorável a regular; ruim a desfavorável $(4,4 \%)$ e nulo a ruim $(1,59 \%)$ (Tabela 2).
Tabela 2. Análise da função sexual medida pelo QS-F das 68 mulheres atendidas em serviço de ginecologia, Goiânia/GO, 2018.

\begin{tabular}{lcc}
\hline Desempenho & $\boldsymbol{N}$ & $\boldsymbol{f ( \% )}$ \\
\hline Bom a Excelente & 18 & 26,5 \\
Regular a Bom & 36 & 52,9 \\
Desfavorável a Regular & 10 & 14,7 \\
Ruim a Desfavorável & 3 & 4,4 \\
Nulo a Ruim & 1 & 1,5 \\
\hline
\end{tabular}

Em relação à análise comparativa dos aspectos demográficos com o Quociente Sexual Feminino foi demonstrado que as mulheres solteiras apresentaram função sexual significativamente superior em relação às casadas $(p=0,048)$ (Tabela 1$)$.

No tocante aos aspectos socioculturais da pesquisa e sua relação com os escores do QS-F, foi constatado que mulheres que referiram que frequentemente iam a bares e festas para encontrar os amigos apresentaram melhor função sexual $(p=0,024)$, bem como aquelas que frequentemente realizavam algum tipo de atividade artística, como ida ao teatro, cinema, sair para dançar, ouvir música e ir a show musical $(p=0,046)$, e afirmaram que frequentemente realizavam atividades turísticas, como passeios pela cidade ou viagens nacionais e internacionais $(p=0,022)$ (Tabela 3).

Tabela 3. Comparação da função sexual com os aspectos socioculturais das 68 mulheres atendidas em serviço de ginecologia, Goiânia/GO, 2018.

\begin{tabular}{|c|c|c|c|c|c|}
\hline Variáveis & $n$ & $f(\%)$ & Média & $D P$ & $p$-valor \\
\hline \multicolumn{6}{|c|}{ Acessa site de relacionamentos } \\
\hline Sim & 12 & 17,6 & 74,2 & 22,3 & \\
\hline Não & 56 & 82,4 & 70,5 & 13,8 & 0,071 \\
\hline \multicolumn{6}{|c|}{ Frequência que realiza as seguintes atividades: } \\
\hline \multicolumn{6}{|l|}{ Grupos Sociais } \\
\hline Frequentemente & 53 & 77,9 & 72,4 & 13,1 & \\
\hline Às vezes & 14 & 20,6 & 66,3 & 22,7 & 0,666 \\
\hline Raramente & 1 & 1,5 & & & \\
\hline \multicolumn{6}{|c|}{ Ida a bares, festas para encontrar amigos } \\
\hline Frequentemente & 32 & 47,1 & 76,1 & 13,1 & \\
\hline
\end{tabular}




$\begin{array}{llllll}\text { Às vezes } & 20 & 29,4 & 69,2 & 14,4 & \\ \text { Raramente } & 16 & 23,5 & 63,8 & 18,5 & \mathbf{0 , 0 2 4}\end{array}$

\section{Atividade Física}

Frequentemente

Às vezes

Raramente

Atividade Artística

Frequentemente

Às vezes

Raramente

Atividade Manual

Frequentemente

Às vezes

Raramente

Atividade Intelectual

$\begin{array}{lccccc}\text { Frequentemente } & 45 & 66,2 & 72,4 & 14,9 & \\ \text { Às vezes } & 18 & 26,5 & 66,9 & 17,7 & \\ \text { Raramente } & 5 & 7,4 & 75,2 & 11,5 & 0,373\end{array}$

Atividade Turística

Frequentemente

Às vezes

Raramente

$\begin{array}{lllll}31 & 45,6 & 75,5 & 11,7 & \\ 15 & 22,1 & 68,9 & 16,4 & \\ 22 & 32,4 & 66,5 & 18,4 & 0,091\end{array}$




\section{Discussão}

A falta de desejo, ausência de orgasmo, distúrbio na excitação, desconforto e/ou dor no ato sexual compõem o quadro de disfunção sexual. A organização Mundial da Saúde já considera a disfunção sexual como um problema de saúde pública, haja vista o forte impacto na qualidade de vida das pessoas acometidas ${ }^{8}$.

No conjunto da amostra do presente estudo, obteve-se uma média do escore total de 71,2 pontos no QS-F, sendo desempenho regular a bom da função sexual. A maioria da amostra se enquadrou nessa classificação. Apesar da escassez de trabalhos sobre o assunto, é estimada uma prevalência de disfunção sexual entre $25 \%$ e $63 \%$ a nível mundial ${ }^{9}$. Outro estudo traz uma incidência entre 20 a $50 \%$ da população feminina10. É fato que a disfunção sexual feminina tem alta prevalência na população, sendo os valores encontrados na presente pesquisa de acordo com tal informação.

Inúmeros fatores podem influenciar na sexualidade feminina, colaborando com o desenvolvimento da disfunção sexual. Eles podem ser divididos em aspectos físicos, psicossociais e sociodemográficos. Segundo estudos, os fatores psicossociais com maior impacto na função sexual feminina são a depressão, a ansiedade, abuso sexual prévio, negligência emocional e estresse excessivo. A imagem corporal negativa também tem forte impacto na sexualidade feminina. Ademais, problemas de relacionamento, contextos culturais ou religiosos inibidores da sexualidade também interferem na função sexual da mulher ${ }^{1,11,12}$.

Pode-se perceber que, para a manutenção de uma boa função sexual feminina, é imprescindível promover a saúde física, mental e uma relação afetiva emocionalmente satisfatória para a mulher. Qualquer tipo de interferência em alguma dessas áreas poderá culminar com alterações na resposta sexual feminina e, consequentemente, na disfunção sexual.

Dentre os fatores demográficos analisados nesse estudo identificou-se uma melhor função sexual em mulheres solteiras em relação às casadas/união estável. Ao contrário do achado no presente estudo, uma pesquisa realizada com mulheres brasileiras identificou que aquelas que estavam solteiras tinham um pior funcionamento sexual quando comparadas àquelas em relacionamentos estáveis, apresentando prevalência significativamente maior de problemas nos domínios de lubrificação, orgasmo, satisfação e dor ${ }^{13}$.

Em relação aos aspectos socioculturais, no presente estudo foi demonstrado que mulheres que frequentemente iam a bares e festas para encontrar os amigos, que realizavam algum tipo de atividade artística, como ida ao teatro, cinema, sair para dançar, ouvir música e ir a show musical e atividades turísticas, como passeios pela cidade ou viagens nacionais e internacionais apresentaram melhor função sexual. Pressupõe-se que um estilo de vida ativo possa influir em uma melhor saúde sexual nas mulheres.

Fatores como depressão, estresse e até mesmo o cansaço excessivo se correlacionam negativamente com a função sexual ${ }^{11}$. Dessa forma, as mulheres que têm uma vida social mais dinâmica e funcional, como as relatadas acima, podem atenuar os fatores estressantes cotidianos e os níveis de cansaço, ansiedade e tristeza, melhorando indiretamente a saúde sexual.

Considerando os aspectos psicossociais, existe um consenso correlacionando a ocorrência de uma maior disfunção sexual em mulheres que possuem algum transtorno afetivo, baseado em estudos dos Estados Unidos, Suíça, França, entre outros. Dentre esses transtornos, o mais importante é a depressão, na qual todas as fases do ciclo de resposta sexual estão alteradas, sendo a ausência de desejo e dores associadas ao ato sexual as complicações mais frequentes ${ }^{14}$. Corroborando com esses dados, identificou-se no estudo que as mulheres que não se consideravam como sendo uma pessoa triste apresentaram função sexual substancialmente melhor comparadas àquelas que se consideravam tristes.

De fato, a relação entre humor depressivo e disfunção sexual é bidirecional, visto que a depressão pode ser um fator de risco para a disfunção sexual, assim como a medicação com antidepressivos pode provocar efeitos sexuais adversos. Além disso, um desempenho sexual insatisfatório também pode ser considerado importante resultante psicossocial, estressor para perda de autoestima, diminuição da libido e levar a depressão. Portanto, não existe uma direção exata da causa das variáveis analisadas, podendo-se comprovar apenas a correlação entre ambas afecções, que costumam ocorrer simultaneamente. Ademais, são consideradas um problema de saúde pública devido à alta prevalência e impacto negativo na qualidade de vida ${ }^{14}$.

Levando em consideração a ingesta do álcool, em nosso estudo obtivemos uma associação positiva: aquelas que faziam ingesta de álcool apresentaram função sexual substancialmente melhor comparadas as que não a faziam. No conjunto dos resultados apresentados, é possível estabelecer uma relação dessas mulheres com uma dinâmica social ativa, inseridas nos contextos socioculturais. Entretanto, na literatura observa-se uma associação negativa entre a ingesta de álcool e a sexualidade, sendo relacionado com os efeitos adversos dele. Tais efeitos são comparados ao uso de droga psicoativas, narcóticos, anticoncepcionais hormonais, antidepressivos e tabagismo ${ }^{14,15}$.

Destaca-se também que transtornos psiquiátricos e fatores subjetivos, como autoimagem, relacionamento conjugal e fatores físicos, como a obesidade, podem afetar a função sexual na 
mulher ${ }^{16}$. Na literatura, entretanto, existe algumas divergências quanto à relação da função sexual com a obesidade. Em um estudo realizado na Universidade Federal do Rio Grande do Norte (UFRN) comparou as diferenças na incidência de disfunção sexual nos seis diferentes domínios de mulheres brasileiras obesas e não obesas. Por meio da utilização do Female Sexual Function Index demonstrou-se não ter diferença significante nas incidências de disfunção sexual feminina entre pacientes obesas e não obesas. Apesar disso, foi evidenciada uma diferença interessante na distinção nos aspectos sexuais alterados nos dois grupos, nas mulheres obesas houve impacto em apenas três domínios subjetivos do Female Sexual Function Index (desejo, orgasmo e excitação), já no grupo controle houve alteração em cinco aspectos: desejo, orgasmo, excitação, dor e lubrificação ${ }^{17}$.

Entretanto, nesse mesmo estudo as disfunções associadas à obesidade foram menos evidenciadas em domínios fisiológicos, sugerindo que aspectos psicológicos pareciam estar primariamente envolvidos na etiologia da disfunção sexual de mulheres em situação de obesidade ${ }^{17}$. Reforçando a ideia apresentada, um resultado importante do presente trabalho foi a evidência de que a função sexual foi significativamente melhor nas mulheres que referiram estar satisfeitas com o próprio corpo, demonstrando que a sexualidade bem vivida é um reflexo da saúde geral.

Diferentemente, um outro estudo cujo objetivo foi de apreender como a mulher com excesso de peso percebe seu corpo e como se relaciona com sua sexualidade, a relação entre obesidade e sexualidade foi vista como conflituosa e negativa, estando essas mulheres vulneráveis a desordens psíquicas, sociais e emocionais. Infere-se que a obesidade é responsável sim pela diminuição da qualidade de vida sexual tanto feminina quanto masculina, porém, não parece constituir um fator de risco independente para uma baixa qualidade de vida sexual feminina. O resultado da magreza como novo ideal de beleza está relacionado com a inversão de valores que aconteceram no século XX da representação de saúde não mais associada com a gordura, sendo o corpo visto como um objeto de caráter histórico que sofre um processo resultado das relações na sociedade ${ }^{18}$.

Nesse sentido, observa-se na contemporaneidade que a sexualidade é reconhecida como um fenômeno complexo e as dificuldades sexuais são influenciadas por inúmeros fatores que podem alterar a qualidade de vida e as relações interpessoais. Além dos já reconhecidos fatores hormonais e biológicos, os fatores psicossociais cada vez mais estão sendo identificados como determinantes na disfunção sexual na mulher.

\section{Conclusão}

É possível verificar nesse estudo que, além das condições biológicas, os preditores psicossociais mostraram-se de grande relevância na identificação de melhor ou pior função sexual na mulher em idade fértil.

Evidenciou-se que mulheres que frequentemente iam a bares e festas, que realizavam algum tipo de atividade artística e atividades apresentaram função sexual significativamente melhor. Em conjunto àquelas que estavam solteiras, que afirmaram fazer ingesta de álcool, que não se consideravam como sendo uma pessoa triste e as que referiram estar satisfeitas com o próprio corpo.

A sexualidade da mulher e o seu disfuncionamento são assuntos de extrema relevância, haja vista o forte impacto que uma má função sexual pode gerar na qualidade de vida da mulher em idade fértil. Conquanto, o tema ainda é pouco explorado pelas pesquisas, sobretudo em relação a população feminina adulta e sem comorbidades clínicas.

Ratifica-se, portanto, a importância de serem realizadas mais pesquisas sobre a função sexual feminina na população adulta e que, aparentemente, gozam de perfeita saúde biológica. É preciso cada vez mais se voltar as atenções para preditores psicossociais de saúde que podem influenciar na função sexual. Essas são causas subjetivas e complexas de serem compreendidas, mas que corroboram substancialmente para o surgimento da disfunção sexual, como foi demonstrado nesse estudo.

\section{Referências}

1. Trindade WR, Ferreira MA. Sexualidade feminina: questões do cotidiano das mulheres. Texto \& Contexto Enfermagem, 2008; 17(3):417-26.

2. Vieira KFL, Nóbrega RPM, Arruda MVS, Veiga PMM. Representação social das relações sexuais: um estudo transgeracional entre mulheres. Psicologia: Ciência e Profissão, 2016; 36(2):329-40.

3. Tozo I, Lima SMRR, Gonçalves N, Moraes JC, Aoki T. Disfunção sexual feminina: a importância do conhecimento e do diagnóstico pelo ginecologista. Revista Arquivos Médicos dos Hospitais e da Faculdade de Ciências Médicas da Santa Casa de São Paulo, 2007; 52(3):94-9.

4. Fleury HJ, Abdo CHN. Tratamento psicoterápico para disfunção sexual feminina. Diagnóstico \& Tratamento, 2012; 17(3):133-7.

5. Lucena BB, Abdo CHN. O papel da ansiedade na (dis) função sexual. Diagnóstico \& Tratamento, 2013; 18(2):94-8.

6. Hochman B, Nahas FX, Oliveira Filho RS, Ferreira LM. Desenhos de pesquisa. Acta Cirúrgica Brasileira, 2005; 20(suppl.2):2-9. 
7. AbdoCHN.Quociente sexual feminino: um questionário brasileiro para avaliar a atividade sexual da mulher. Diagnóstico \& Tratamento, 2009; 14(2):89-91.

8. Bomfim IQM, Batista RPS, Lima RMC. Avaliação da função sexual em um grupo de mastectomizadas. Revista Brasileira de Promoção à Saúde, 2014; 27(1):77-84.

9. Ribeiro B, Magalhães AT, Mota I. Female sexual dysfunction in the reproductive years: prevalence and associated factors. Revista Portuguesa de Medicina Geral e Familiar, 2013; 29(1):16-24.

10. Prado DS, Mota VPLP, Lima TIA. Prevalência de disfunção sexual em dois grupos de mulheres de diferentes níveis socioeconómicos. Revista Brasileira de Ginecologia e Obstetrícia, 2010; 32(3):139-43.

11. Enomoto SM, Pires IAL, Rizzi SKLA, Haddad CAS, Nazário ACP, Facina G. Avaliação da imagem corporal e da função sexual em mulheres com linfedema após tratamento cirúrgico de câncer de mama. Revista Brasileira de Mastologia, 2014; 24(1):9-16.

12. Faubion SS, Rullo JE. Disfunção sexual na mulher: uma abordagem prática. Revista Portuguesa de Medicina Geral e Família, 2015; 31:351-3.

13. Pereira VM, Nardi AE, Silva AC. Sexual dysfunction, depression, and anxiety in young women according to relationship status: an online survey. Trends of Psychiatry Psychother, 2013; 35(1):55-61.

14. Lucena BB, Abdo CHN. Considerações sobre a disfunção sexual feminina e a depressão. Diagnóstico \& Tratamento, 2012; 17(2):82-5.

15. Lara LAS, Rosa e Silva ACJS, Romão APMS, Junqueira FRR. Abordagem das disfunções sexuais femininas. Rev. Bras. Ginecol. Obstet. 2008; 30(6):312-21.

16. Bedone RMV, Abdo CHN. Síndrome metabólica como fator de risco para disfunção sexual feminina. Diagnóstico \& Tratamento, 2013; 18(1):45-48.

17. Carrilho PJF, Vivacqua CA, Godoy EP, Bruno SS, Brígido $A S$, Barros EF, et al. Sexual dysfunction in obese women is more affected by psychological domains than that of non-obese. Rev. Bras. Ginecol. Obstet.2015; 37(12):552-8.

18. Ribeiro RG, KruseMHL. O corpo da mulher em revista: o imperativo da beleza. Texto Contexto Enfermagem, 2014; 23(1):101-8. 